Synthesis, vol. 25 no 1, e028, junio 2018. ISSN 1851-779X

Universidad Nacional de La Plata.

Facultad de Humanidades y Ciencias de la Educación.

Synthesis

Centro de Estudios Helénicos

\title{
Consideraciones preliminares para un nuevo modelo teórico de la métrica griega antigua: el caso del verso eólico
}

\author{
Alejandro Abritta * \\ * Universidad de Buenos Aires - CONICET, Argentina
}

Cita sugerida: Abritta, A. (2018). Consideraciones preliminares para un nuevo modelo teórico de la métrica griega antigua: el caso del verso eólico. Synthesis, 25(1), e028. https://doi.org/10.24215/1851779Xe028 


\title{
Consideraciones preliminares para un nuevo modelo teórico de la métrica griega antigua: el caso del verso eólico
}

\author{
Preliminary Considerations for a new Theoretical Model of Ancient Greek Metre: the Case of Eolic Verse \\ Alejandro Abritta * \\ Universidad de Buenos Aires - CONICET, Argentina
}

\section{RESUMEN:}

El objetivo de este trabajo es realizar algunas consideraciones teóricas sobre el metro griego, con atención particular al estudio del verso de los poetas eólicos (Safo y Alceo). Se analizan algunos de los modelos teóricos vigentes para su estudio, intentando mostrar sus limitaciones y la necesidad de elaborar un nuevo modelo que resulte más adecuado para la comprensión del objeto de estudio. Asimismo, se busca demostrar que el modelo presentado por Dale (1969: 41-97) y ampliado por Sicking (1993) es el que con mayor precisión y simpleza describe las estructuras formales del verso griego, de modo que puede proveer una base para su descripción de nivel abstracto. Se argumenta, por otra parte, que es necesario complementar esa descripción con un análisis del nivel de la realización, apoyado fundamentalmente en el estudio cuantitativo de los poemas.

Palabras clave: Métrica griega, Verso eólico, Safo, Alceo.

\section{Abstract:}

The goal of this paper is to present some theoretical considerations about Greek meter, with special attention to the study of the verse of the eolic poets (Sappho and Alcaeus). We analyze some of the current theoretical models for its study, trying to show their limitations and the need for a new model, more suitable for understanding the object of study. Likewise, we seek to demonstrate that the model presented by Dale (1969:41-97) and extended by Sicking (1993) is the one that with greater precision and simplicity describes the formal structures of the Greek verse, and therefore it can offer a basis for its abstract level description. We argue, on the other hand, that it is necessary to complement that description with an analysis of the realization level, based fundamentally in the quantitative study of poems.

KEYWORDS: Greek meter, Eolic verse, Sappho, Alcaeus.

\section{INTRODUCCIÓN}

Como sucede en muchas áreas de la ciencia, las discusiones teóricas sobre el análisis formal del verso griego antiguo suelen parecer diálogos de sordos. En su extenso trabajo sobre el verso pindárico, Itsumi (2011: 3-7) no ahorra críticas a un enfoque presentado por Dale (1969: 41-97), llegando a la conclusión (p. 21) de que "la notación (...) de Dale busca excluir consideraciones de tipología métrica y es, por esa razón, comparativamente poco informativa". Esta observación, no obstante, solo es válida en un marco teórico en el que "tipología métrica" implica la aparición de cola similares en diferentes autores o, en otras palabras, el principio metodológico de que la única manera de asociar diferentes esquemas métricos es que todos tengan las mismas etiquetas. Decir que una teoría que excluye consideraciones colométricas es "poco informativa" solo puede ser cierto, entonces, en un marco teórico que entiende que "informar" sobre los metros es clasificarlos aplicándoles esas etiquetas. ${ }^{1}$

Es importante notar que esto no implica bajo ningún concepto que la metodología de trabajo de Itsumi esté equivocada. No es ese el inconveniente que intento destacar. El problema es la falta de conciencia del autor sobre el hecho de que su modelo de análisis no es una reconstrucción de una realidad del verso, sino una herramienta teórica para aproximarse a él. La diferencia no es menor, porque los criterios de evaluación en un caso y en otro divergen. Si quisiéramos un modelo del verso griego tal y como lo entendieron sus compositores, la principal evidencia que deberíamos utilizar es el testimonio de los antiguos. ${ }^{2}$ Pero una herramienta teórica para el análisis del verso se evalúa sobre la base de otros criterios: su poder explicativo, su simplicidad, etc. Para dejarlo en claro: concebir nuestras teorías sobre el metro griego como herramientas teóricas para su análisis es afirmar que su objeto de estudio no es el "proceso compositivo" del verso sino el propio verso. En qué 
medida lo que elaboremos coincida con el modo en que los poetas de hecho componían es intrascendente y, más importante, incomprobable.

Aunque esto debería resultar relativamente obvio para cualquiera que se aproxime al análisis de la poesía con un cierto grado de conocimiento de su estado de conservación y la insuficiencia de la evidencia sobre su contexto cultural, las discusiones en metricología tienden a centrarse más en la búsqueda de la "verdad" de los metros que en la elaboración de modelos teóricos adecuados. ${ }^{3}$ Hace unos años, Silva Barris (2011) presentó un estudio integral de la poesía basado en la idea de que su análisis debía concentrarse en la exploración de modelos de escansión, de modo que el eje de la discusión consistiría en la determinación exacta de los valores temporales de cada ubicación métrica. Es el último (por ahora) ejemplo de un modelo duracional del verso, cuya historia se remonta al siglo XIX. ${ }^{4}$ Se ha discutido mucho sobre la validez de este tipo de teorías, pero, notablemente, no se ha introducido en el debate un aspecto fundamental: quién tiene la carga de la prueba. En otras palabras, si un fenómeno métrico x puede ser explicado por una presión duracional y por una tendencia rítmica en un marco tradicional, ${ }^{5}$ ¿qué alternativa es preferible a priori?

El objetivo de este trabajo es, como indica su título, realizar algunas consideraciones preliminares de este tipo, concentrándose en el problema del análisis del verso eólico de Safo y Alceo (esto es, en esta etapa, no en los versos "eólicos" de Píndaro, Baquílides y los trágicos, aunque realizaré unas pocas observaciones a partir del estudio de los versos estíquicos más importantes). Es evidente que para un asunto de la complejidad del presente la restricción es necesaria, pero al llegar a las conclusiones me propongo haber dejado claro que existen motivos suficientes para adherir a un modelo teórico en la línea del propuesto por Sicking (1993), con una serie de consideraciones adicionales necesarias para convertir un esquema esencialmente descriptivo en un modelo teórico con potencial explicativo de los distintos fenómenos del verso griego. Asimismo, un elemento que, hasta donde sé, nunca ha sido incluido en el debate será importante en lo que sigue: cualquier modelo que se elabore sobre el metro griego no solo debe ayudarnos a entenderlo, sino también ser accesible para los estudiantes y estudiosos de la Antigüedad, que pocas veces, si alguna, incorporan aspectos de análisis métrico en su trabajo. ${ }^{6}$ Esta deficiencia que los metricólogos acaso tenderían a atribuirles es en realidad propia de las teorías métricas, cuya dificultad e inaccesibilidad hacen imposible que las utilicen los no-especialistas.

\section{DE QUÉ TRATA UNA TEORÍA SOBRE EL VERSO GRIEgo}

¿Qué debe informarnos un modelo teórico sobre el verso eólico? La pregunta parece sencilla, pero es bastante difícil de responder. La primera respuesta intuitiva que uno podría dar es “cómo es el verso eólico", esto es, la teoría debería ofrecernos una descripción de su objeto de estudio. Hay dos motivos por los cuales esto no puede ser correcto.

En primer lugar, porque si ese fuera el caso, podríamos decir que una teoría que tuviera como premisa "el verso griego se compone de sucesiones de sílabas largas y breves" y "el primer verso de la estrofa sáfica puede

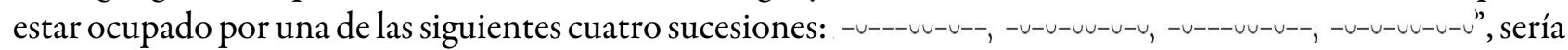
una teoría admisible sobre el objeto de estudio. Pero es evidente que esto no puede ser así. Ese modelo es incapaz de ofrecernos información esencial, como por ejemplo que la cuarta ubicación y la última no son iguales al resto. Además, si se aplicara en otros casos, "explicaría” al hexámetro como un verso que puede estar ocupado por una de 64 posibles sucesiones.

El segundo motivo por el cual una teoría sobre el verso eólico no puede ser solo una respuesta a "cómo era el verso eólico" es que una respuesta a esa pregunta no tiene por qué decir nada de qué rasgos caracterizan en particular a ese tipo de metros. En otras palabras, aunque las premisas del párrafo anterior pueden informar sobre las formas posibles del endecasílabo sáfico, no dicen nada de por qué una sucesión tal debe ser considerada un "verso eólico". 
Por lo tanto, el modelo teórico que se está tratando de desarrollar no puede ser meramente descriptivo, necesita tener reglas en diferentes niveles que permitan, por un lado, facilitar la descripción de los metros y, por el otro, identificar las características de los géneros compositivos.

Pero estos objetivos tampoco bastan, porque el verso griego se compone de dos tipos de elementos de naturaleza por completo diferentes: las palabras y las sílabas. ${ }^{7}$ La distribución de las primeras y de las segundas sigue reglas distintas. Cualquier modelo teórico que se adopte debe ser capaz de dar cuenta de esta realidad. Así, en el caso del tercer verso de la estrofa sáfica, no es suficiente señalar que las sílabas se distribuyen en él en una sucesión $-v-x-v v-v-x-v v-x$ (introduciendo ya el signo de anceps para las ubicaciones que pueden ser ocupadas por una sílaba larga o una breve), sino que es necesario indicar $e$. g. que después del segundo

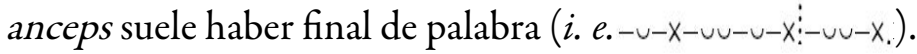

La introducción del elemento anceps en el nivel silábico trae a la luz un problema adicional, el de la realización efectiva de ese tipo de elementos en el verso. En otras palabras, mientras que las premisas hipotéticas dadas al comienzo de esta sección pueden aplicarse sin más a las líneas concretas, la aparición de una ubicación que admite diferentes realizaciones implica la distinción entre dos niveles: el del análisis abstracto, donde existe el elemento anceps, y el de los versos efectivos, donde ese tipo de elemento es imposible. ${ }^{8}$

Y esto último lleva a otro inconveniente que un modelo métrico debe afrontar: cuáles son los elementos de nivel abstracto que es necesario tomar en cuenta en el análisis. Un enfoque tradicional responderá "las sílabas largas y las sílabas breves", pero los duracionalistas proponen algo completamente distinto. En un trabajo como el de West (1970), el nivel de la descripción abstracta está compuesto en forma exclusiva de elementos indefinidos. $^{9}$

Por otra parte, existe la posibilidad de concebir un sistema de elementos para el nivel abstracto y otro para el nivel de la realización. Se podría sostener que un longum es un elemento de nivel abstracto que en el de la realización puede ser ocupado por diferentes tipos de sílabas. Un modelo teórico de este tipo limita la cantidad de elementos fundamentales pero multiplica sus realizaciones posibles.

Sistematizando estas exigencias, un modelo teórico sobre el verso eólico debe ser capaz de dar cuenta de 1) qué tipo de elementos de nivel abstracto existen en el metro, 2) qué tipo de elementos del nivel de la realización existen en el metro, 3) cómo se rige la relación entre los dos niveles y 4) qué rasgos (de los incluidos en los puntos 1 a 3) distinguen al verso eólico como género compositivo.

A estas exigencias “internas" es posible añadir una "externa”, estándar para cualquier teoría científica: el modelo debe tender a ser lo más simple posible, de modo que su aplicación y enseñanza sea accesible para los investigadores y estudiantes de la disciplina. Esta obviedad aparente, como cualquiera que haya recorrido algunos manuales de métrica sabe, ha sido ignorada por los filólogos en forma sistemática.

\section{EL MODELO ESTÁNDAR}

Actualmente, la teoría dominante sobre el verso griego es una que se puede denominar "colométrica". Aunque, como observa García Novo (2008: 400-401), el concepto de elementa de Maas (1962: 23-32) se ha convertido en un estándar en la disciplina, un examen detenido de las descripciones teóricas de los metros permite detectar que ese concepto es apenas auxiliar en la mayoría de ellas. El eje conceptual de los análisis contemporáneos es el colon, esto es, una unidad mínima de elementa que se combina con otras para formar secuencias más largas. ${ }^{10}$

Los modelos teóricos colométricos consisten siempre en una ley de la forma "si $x$ es verso A, entonces $x$ es combinación de colon $\mathrm{a}+$ colon $\mathrm{b}+\ldots$... En la práctica, esto ha llevado a discusiones de dos tipos: qué combinaciones corresponden a qué versos y qué procedimientos de combinación y modificación de cola son admisibles. Estas discusiones, a su vez, han derivado en la elaboración de teorías en donde una complejidad 
enorme es la única constante, pero en las que varían dos factores en proporción inversa: a mayor cantidad de cola, menor cantidad de procedimientos se admiten y, por supuesto, viceversa.

El caso del verso eólico es prototípico de los debates colométricos. Hasta la aparición de Snell (1982 [1955]), las discusiones estándar son las que ejemplifica un trabajo como el de Irigoin (1956). ${ }^{11}$ El problema fundamental con el que lidia el artículo es qué cola componen los versos de las estrofas alcaica y sáfica, y la metodología para resolver esto se basa en dos premisas, la segunda de las cuales caracteriza el enfoque de Irigoin: primero, los cola se identifican a partir del análisis de la distribución de palabras y, segundo, el límite de los cola no coincide con las ubicaciones con final de palabra frecuente.

La propuesta de Snell produjo una inversión en la proporción entre "procedimientos" y "cantidad de combinaciones" mencionada arriba. Para el autor, los versos eólicos están compuestos a partir de dos principios, la "expansión interna" y la "expansión externa”. A una serie de cola fundamentales, e. g. el gliconio $(=g l=x \mathrm{x}-\cup v-\cup \mathrm{x})$, se los puede expandir añadiendo antes de la secuencia de cierre "lkx" un coriambo (- $\left.v_{-}\right) \mathrm{o}$ un dáctilo (-vv) dando lugar a estructuras como $g l^{d}$ ( $\left.=x x-v v-v v-u x\right)$ o $g l^{c}$ (=xx-vu--uv-u-). Este proceso es el que se denomina "expansión interna". Al mismo tiempo, es posible combinar dos de los cola fundamentales o añadirles antes o/y después de la estructura principal un yambo (x-v-) o un yambo "abreviado", i. e. un crético (- - ) o un baqueo( ( --). Este segundo mecanismo es el llamado "expansión externa”.

Si esto puede resultar difícil de asir en una primera aproximación, se vuelve mucho más complejo cuando se observa que los procedimientos de expansión mencionados son los superficiales, dado que existen tres más que permiten "explicar" cómo un colon fundamental se convierte en otro: la "acefalía", la "catalexis" y la "hipercatalexis". Por el primero, a un colon se le remueve la primera sílaba, de modo que un gliconio se convierte en un telesileo $(=p g l=t l=x-v v-v x)$; por el segundo, a un colon se le remueve la última sílaba, de modo que un gliconio se convierte en un ferecracio $(=$ pher $=x \mathrm{x}-\mathrm{v} v-\mathrm{x})$; por el tercero, a un colon se le agrega una sílaba al final, de modo que un gliconio se convierte en un hiponacteo ( $=$ hipp $=\mathrm{xx}-\mathrm{v} v-\mathrm{v}-\mathrm{x}$ ). Por supuesto, un colon puede ser acefálico y cataléctico $(e . g$. el gliconio acefálico cataléctico, el reiziano $=r=$ $\mathrm{X}-\mathrm{v}-\mathrm{x}$ ) y a su vez expandirse interna y externamente (e.g. - $-\mathrm{-}-\mathrm{X}-\mathrm{v}-\mathrm{v} v-\mathrm{v}-\mathrm{X}=\operatorname{cr} p h i p p^{d}$, uno de los dos posibles

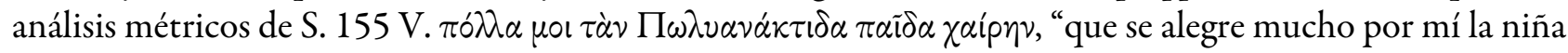
polianáctida").

La lógica del análisis de Snell resulta atractiva quizás porque da la sensación de que explica todas las estructuras conservadas con una cantidad restringida de conceptos. Si uno deja de lado los muchos nombres heredados o inventados para los cola, basta con las nociones de "expansión interna", "expansión externa", "acefalía”, "catalexis" e "hipercatalexis", más un colon fundamental (el gliconio), para dar cuenta de todas las formas del verso eólico. Un corpus de cierta complejidad se simplifica bastante de esta manera. No obstante, este atractivo se sostiene sobre una falacia muy evidente pero, por razones difíciles de explicar, ignorada por la mayoría de los investigadores: el modelo de Snell deja fuera de la categoría de "versos eólicos" una parte más que considerable de los versos conservados de los poetas eólicos. En otras palabras, los poetas eólicos compusieron una parte de su obra en versos eólicos y otra en versos no-eólicos. ${ }^{12}$

Como con la afirmación de Itsumi sobre Dale citada al comienzo de este trabajo, esto no significa que la teoría esté equivocada. Es obviamente admisible que un poeta componga en más de un estilo. El problema acuciante de aceptar esto, no obstante, es que la cuestión 4 mencionada al final de la sección anterior pasa a resolverse con una petitio principii, a saber, los versos eólicos son los que la teoría considera "versos eólicos" y no los versos de los poetas eólicos. Los rasgos que los caracterizan, sin duda, los distinguen del resto de los metros griegos, pero lo hacen a priori, no sobre la base de un estudio de la evidencia conservada en el corpus de poemas de origen eólico.

Ese, sin embargo, es solo el primero de los inconvenientes, porque otros empiezan a acumularse cuando se confronta el modelo de Snell con el resto de las exigencias. Aunque el mecanismo de elaboración de metros propuesto por el autor tiene su elegancia, de hecho no responde a prácticamente ninguna de las preguntas presentadas por ellas. Parece claro que los elementos de nivel abstracto son los cola, pero no lo es tanto 
cuáles. ¿Solo el gliconio? ¿El gliconio, el ferecracio y el hiponacteo? ¿El gliconio, el ferecracio, el hiponacteo, el telesileo, el reiziano y el "octosílabo" que West (1982: 30) llama hagesichorean y es la forma acefálica del hiponacteo o, acaso, los 39 períodos que Snell (1982: 45) registra como atestiguados? ¿O los 42 períodos posibles producto de la expansión interna más el yambo, ${ }^{13}$ el crético y el baqueo? ¿O los 17 que menciona West (1982: 30-31)?

La respuesta más plausible es que todas las opciones son correctas y que la teoría colométrica es en realidad un complejo sistema para la descripción del nivel abstracto de los metros. ${ }^{14}$ Esto puede hallar una confirmación de cierto tipo en el hecho de que, desde el trabajo de Snell, todo interés por el nivel de la realización de los versos eólicos parece haberse agotado y las únicas preocupaciones que la metricología suele expresar son si un determinado verso es una forma acefálica, una forma expandida, una combinación de cola, etc. La única excepción importante se halla en los enfoques duracionalistas, pero incluso en ellos las cuestiones no son del nivel de la realización, sino del de los esquemas. En efecto, preguntarse si una determinada ubicación tiene una duración de larga o de breve no es encargarse de un problema de la realización del verso, sino de su descripción en el nivel abstracto. ${ }^{15}$

Un segundo síntoma de esta tendencia a la formalización se manifiesta en un proceso de "colapso" de niveles métricos que ha sido una constante desde el esfuerzo de Maas por simplificar el abigarrado campo de la metricología. Diferentes autores han elevado al nivel de los esquemas abstractos distintos aspectos del verso, siendo una constante la incorporación de los finales de palabra frecuentes bajo el concepto de "cesura" y su evasión bajo el concepto de "puente". También la realización de los diferentes elementos (e.g. en el uso del signo ãã para un par de anceps que no pueden ser realizados ambos con sílaba breve) y, por supuesto, las "duraciones silábicas" admitidas han sido consideradas parte esencial de los esquemas, generando no solo una diversidad notable de enfoques de análisis sino también una serie de teorías confusas y complejas que dificultan el estudio del verso al punto de que, con escasas excepciones, este ha sido abandonado en la investigación filológica no metricológica casi del todo.

Este resultado se parece en cierta medida al alcanzado por la astronomía ptolemaica: la elegancia inicial de un enfoque poderoso termina por sacrificarse en una revisión permanente de las deficiencias en pos de una adecuación a la evidencia que siempre se puede alcanzar pero nunca sin avanzar un paso más en hacer más compleja la teoría. ${ }^{16}$ Hoy la colometría es capaz de dar cuenta de, virtualmente, todos los metros griegos, pero no sin tener que tolerar una infinidad de casos de ambigüedad de análisis ni tampoco sin haber abandonado, por completo, toda pretensión de simplicidad teórica. ${ }^{17}$ Esto explica que, desde hace algo más de treinta años, posiciones alternativas hayan comenzado a presentarse.

\section{ENFOQUES NO COLOMÉTRICOS}

Entre 1950 y 1951, en una serie de tres artículos, A. M. Dale presentó un modelo de análisis de la lírica griega que pretendía superar la dificultad del colométrico, que para ella era aplicable sin dificultades en los coros dramáticos pero no así en la poesía de Píndaro, Simónides, Baquílides, etc. ${ }^{18}$ La autora proponía, sobre la base del análisis maasiano del dáctilo-epítrito, ${ }^{19}$ dejar de lado los cola y concentrarse en las unidades básicas que podían formarse a partir de los elementos del metro griego: las sílabas largas y las sílabas breves. Dado el principio fundamental de la métrica griega de que, en ningún esquema, una distribución de más de dos breves es admisible y la noción de que una secuencia rítmica mínima requiere una repetición, en particular en este caso la repetición del elemento dominante (la sílaba larga), Dale propuso que las unidades básicas debían ser la simple short $\left(=\mathrm{s}=-{ }_{-}\right)$y la double short $\left(=\mathrm{d}=-v_{-}\right)$, esto es, las dos estructuras mínimas posibles que incluyen dos longa. ' lodos los metros líricos debían analizarse a partir de estas unidades y los procedimientos a los cuales estas pueden someterse. 
Resulta razonable atribuir el rechazo a la teoría de Dale a dos factores: en primer lugar, el grado de desarrollo de la colometría, que todavía tenía un largo camino por recorrer antes de llegar al punto de complejidad inmanejable en el que estamos hoy. En segundo lugar, el modo de presentación de la propia autora que, más que sugerir que su propuesta era superadora de los análisis previos, parece implicar que es producto de la resignada aceptación de que algunos metros griegos no se someten con tranquilidad al encasillamiento en cola.

Algo más de cuarenta años después, sin embargo, la situación era diferente. La colometría ya se había demostrado incapaz de resolver muchas de las dificultades que sus partidarios debieron encarar y el enfoque de Dale, por lo tanto, dejó de ser una alternativa triste a la metricología "correcta" y pasó a ser una alternativa teórica viable a una que cada vez más parecía inadecuada. Este es el proyecto que emprende Sicking (1993).

En la siguiente sección me detendré en el modelo de Dale-Sicking, pero antes resulta importante revisar otros dos, más recientes, para descartarlos como alternativas posibles al colométrico. En primer lugar, los trabajos de Golston y Riad (2000 y 2005). Los autores proponen estudiar el verso griego sobre la base de la noción de pie (fonético) y de dos restricciones básicas: ${ }^{20}$ NoCLASH (la prohibición de dos sílabas estresadas contiguas) ${ }^{21}$ y NoLAPSE (la prohibición de moras estresadas contiguas). ${ }^{22}$ El único tipo de metro que no viola ninguna de las dos y, por lo tanto, el único "rítmico", es el anapesto, mientras que el dáctilo se define como la secuencia que viola siempre NoCLASH y el troqueo como la secuencia que viola siempre NoLAPSE.

Mientras que el análisis de las principales formas estíquicas conservadas parece responder bien a este enfoque, su aplicación a los metros líricos resulta mucho menos atractiva. Para poder dar cuenta de este tipo de versos, los autores deben, en su trabajo de 2005, reconvertir las restricciones que, en el de 2000, daban cuenta de la totalidad de las estructuras estíquicas en "primitivos" que pueden combinarse entre sí. El endecasílabo sáfico, por ejemplo, se describe como una estructura con la siguiente forma: LAPSE-CLASH-LAPSE. Esta idea no resulta demasiado atractiva si se toma en cuenta que NoCLASH es una restricción de nivel silábico y NoLAPSE, una restricción de nivel moraico que, si por separado ofrecen una elegante explicación de la oposición entre metros dactílicos y metros yambo-trocaicos, cuando se combinan terminan por no ser más que unidades de análisis equivalentes a las s y $\mathrm{d}$ de Dale, pero más complejas de usar. ${ }^{23}$

En una línea de trabajo completamente diferente, García Novo (1995, 2000, 2008 y 2014) ha presentado otro modelo teórico, "estructural", alternativo al colométrico. La propuesta de la autora es sencilla: abandonar toda unidad diferente a los elementos fundamentales, que para ella son cuatro, un disilábico potencial ( $=\mathrm{D}$ $=\underline{\underline{w}})$ y tres monosilábicos $\left(\mathrm{M}^{\longleftarrow}=\left\llcorner, \mathrm{M}^{-}=-\mathrm{y} \mathrm{M}^{-}=\mathrm{x}\right)\right.$. Este modelo ha sido aplicado por Laguna Ortiz (1997) al verso eólico, con un resultado casi idéntico al modelo de Raven (1962) basado en un "núcleo coriámbico" con componentes a la izquierda y la derecha. Más allá de las deficiencias descriptivas de estos dos trabajos, ${ }^{24}$ la teoría de García Novo resulta poco atractiva por el sencillo motivo de que convierte los elementos fundamentales en las unidades de análisis, resignando de hecho toda posibilidad de producir esquemas métricos que sean algo más que sucesiones de sílabas. Además, y a pesar de la importante observación de que es necesario dejar de lado aspectos de la realización de los versos en su análisis abstracto, colapsa en su elemento disilábico potencial dos tipos de elementos evidentemente diferentes por su comportamiento métrico: los doble-brevia del hexámetro y los longa del trímetro yámbico. ${ }^{25}$

A pesar de lo inadecuado del modelo, ${ }^{26}$ las razones que García Novo presenta para elaborarlo son atendibles y expresan la necesidad real de un nuevo enfoque sobre la métrica griega: los modelos colométricos ignoran la división entre un nivel abstracto y uno de la realización de la poesía y, lo que es más, se detienen en forma excesiva en discusiones sobre componentes que no tienen ningún tipo de realidad en el verso. 


\section{Preliminares de un nuevo modelo sobre el metro griego}

El enfoque inaugurado por Dale y expandido por Sicking a la totalidad de la poesía griega se basa, como ya se ha mencionado, en la identificación de dos unidades básicas. En lo que sigue, sin embargo, reconstruiré y reformularé (hasta cierto punto) la propuesta, partiendo del nivel más alto de abstracción y siguiendo el recorrido que plantean las exigencias introducidas en la sección 2.

La primera pregunta es qué tipo de elementos de nivel abstracto existen en el metro griego. A las respuestas que con razón se darían a esto, querría imponer una primera consideración: si el objetivo es construir una teoría adecuada, lo fundamental que hay que decir es que el elemento más básico es la "ubicación métrica", es decir, la ubicación vacía de contenido que debe ser ocupada por un elemento. Este concepto es necesario por la simple razón de que un metro es, antes que cualquier otra cosa y "translingüísticamente", una sucesión de ubicaciones que deben ser ocupadas por algún tipo de elemento. ${ }^{27}$

Ahora bien, la introducción del concepto de "ubicación métrica" no responde la pregunta formulada de manera satisfactoria; solo permite reformularla. En vez de preguntar qué elementos de nivel abstracto existen, es necesario preguntar qué elementos de nivel abstracto pueden ocupar las ubicaciones métricas. La respuesta mínima concebible es "dos": las sílabas largas y las sílabas breves. No obstante, nadie, hasta donde he podido verificar, admitiría seriamente que esos son los únicos elementos que se necesitan para describir los metros, por la simple razón de que existen ubicaciones en las que, en el nivel de la realización, se observan tanto sílabas largas como sílabas breves, de modo que o bien se admite la coexistencia de esquemas alternantes, ${ }^{28}$ o bien se admite la posibilidad de elementos de nivel abstracto que admiten más de una realización. Se introduce así un tercer elemento, el anceps (pl. ancipitia). ${ }^{29}$

$\mathrm{Si}$, para distinguirlos de los elementos del nivel de la realización, se utiliza longum (pl. longa) para las ubicaciones métricas realizadas por sílabas largas y breve (pl. brevia) para ubicaciones métricas realizadas por sílabas breves, entonces una teoría sobre el verso griego debería contar mínimamente con tres elementos fundamentales: longa, brevia y ancipitia. Ya desde Maas (1962: 25) se admite, sin embargo, la necesidad de un cuarto elemento, el biceps o, el término preferido aquí, doble-breve, que es la ubicación métrica realizada por dos sílabas breves. La inclusión de este elemento está justificada por tres hechos: la frecuencia de metros basados en sucesiones del tipo -uv-, la alternancia regular entre -uv- y --- (que implicaría, de no existir el elemento doble-breve, o bien el reemplazo de dos ubicaciones métricas por una o bien aceptar que una sílaba larga puede ocupar dos ubicaciones métricas, ambas posibilidades con consecuencias devastadoras para la teoría) y la ventaja metodológica de que, gracias al doble-breve, casi todos los metros respetan el principio de que no puede haber dos ubicaciones no-marcadas seguidas. ${ }^{30}$

Los enfoques duracionalistas sugieren que estos cuatro elementos no son suficientes e incluso que no son adecuados, puesto que, por un lado, los esquemas métricos son sensibles a diferencias cuantitativas distintas a la oposición dual larga/breve y, por el otro, una parte importante de la poesía griega era en realidad música con valores rítmicos múltiples. Más allá de la discutible veracidad de estas observaciones, ${ }^{31}$ debe considerarse que se apoyan en una premisa falaz: que nuestro modelo teórico sobre el metro griego debe reflejar la realidad del proceso compositivo en la mente de los poetas. Pero esto es y debe ser falso por una razón sencilla: no tenemos acceso de ningún tipo, ni directo ni mediato, a la realidad del proceso compositivo en la mente de los poetas. Las teorías que se elaboran hoy deben ser evaluadas sobre la base de su poder descriptivo y explicativo y de su simplicidad: incorporar criterios "duracionales", incluso si con ello se ganara algún potencial explicativo (y esto es por demás debatible), implica sacrificar de manera contundente la simplicidad y la capacidad descriptiva de la teoría. ${ }^{32}$

En el nivel de los elementos fundamentales, por lo tanto, bastan los cuatro introducidos: longum, breve, doble-breve y anceps. Como ha observado Dale, sin embargo, para describir los metros se necesita un nivel adicional, porque estos elementos no se presentan en sucesiones arbitrarias, sino ordenados en unidades 
básicas de dos tipos: $\mathrm{s}$ y d. Estas unidades pueden yuxtaponerse, esto es, sucederse unas a otras en su forma completa $\left(e . g \cdot s^{\prime} s=-v--{ }^{-}\right)$o prolongarse, esto es, combinarse para formar unidades más largas (que también pueden yuxtaponerse o prolongarse). La prolongación, además, puede darse de manera directa (e.g. dds $=$

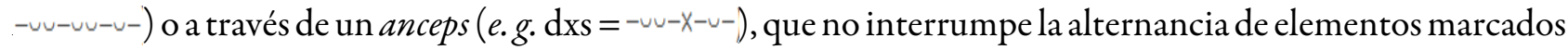
y no-marcados. Nótese, no obstante, que la realización larga de un anceps separa las unidades prolongadas de una forma que no lo hace la prolongación directa, de modo que, en muchos casos, la prolongación por medio de anceps puede servir para delimitar unidades estructurales de la misma forma que la yuxtaposición.

Existe otro grupo de componentes en el nivel abstracto: aquellos que habilitan realizaciones alternativas de los elementos, las "divergencias rítmicas". ${ }^{33}$ La introducción de estas divergencias en un esquema métrico indica la posibilidad de una realización alternativa de un elemento: la "contracción" permite la realización de un doble-breve con una sílaba larga, la "resolución", la realización de un longum con dos sílabas breves y la “colosis”, la realización de un breve con una sílaba larga. ${ }^{34}$

Finalmente, un aspecto clave en el análisis métrico es la unidad central organizativa: el verso. Aunque este concepto requiere una serie de consideraciones especiales, a los fines de la presente exposición el rasgo más importante a destacar es que al final de cada verso existe una ubicación especial, asimilable a un anceps en el hecho de que siempre puede ser ocupada por una sílaba larga o por una sílaba breve. ${ }^{35}$ Asimismo, el final de una secuencia métrica (es decir, de un verso) presenta en todos los géneros poéticos conservados la posibilidad de alternar entre finales "sordos", esto es, los que terminan junto con una unidad básica ( $e . g . x d s=x-\backsim v-\backsim x)$, y finales "sonoros", es decir, a los que se añade un elemento variable más en el cierre (e.g. $x d s x=x-u v-v-x)$. Resulta claro que los poetas se dieron esta libertad para poder contar con dos alternativas rítmicas en el final de cada verso, pero se requiere un estudio detenido sobre la cuestión.Este sistema permite la descripción de cualquiera de los metros griegos conservados. ${ }^{36}$ La estrofa sáfica, por ejemplo, puede caracterizarse de la siguiente manera:

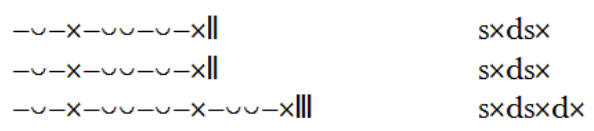

La estrofa alcaica, así:

$$
\begin{aligned}
& x-v-x-v v-v x \| \\
& x-v-x-v v-v x \| \quad \quad x s \times d s \\
& x-v-x-v-x-v v-v v-v-x|l| \quad \quad x \operatorname{s} \times s \times d d s \times
\end{aligned}
$$

Un metro como el usualmente denominado $p h i p p^{2 c}$ o "hiponacteo acefálico con doble expansión coriámbica”, en el que, según Hefestión (10.6 34 C.), estaba escrito el libro tercero de poemas de Safo, cuya estructura es $x-v v--v v--v v--v-x$, se describe $\times d$ 'd'ds $x$. Nótese, además, que el análisis de la notación permite detectar las afinidades entre la mayoría de las estructuras presentadas, que terminan en general con la secuencia ds $(\mathrm{x})$.

La larga respuesta a la primera de las preguntas planteada en la sección 2 facilita responder a las otras. Delimitados los elementos y unidades de nivel abstracto, los de la realización se revelan como los más evidentes: las sílabas en las que se efectúan los longa, brevia, doble-brevia y ancipitia y las palabras. ${ }^{37} \mathrm{El}$ resto de las cuestiones implica un esquema de análisis basado en el estudio estadístico del verso. ${ }^{38}$ Las realizaciones silábicas de los elementos y la ubicación de las palabras no son, obviamente, aspectos de nivel abstracto, sino que dependen del foco y de la muestra que se seleccionen. Así, mientras que un gliconio ${ }^{39}$ (xds) puede estar sometido a una serie de regulaciones particulares en Safo, no lo estará en Alceo o en Baquílides. El esquema abstracto es el mismo y puede reconocerse en todos los autores (lo que hace absurda la afirmación de Itsumi citada al comienzo), pero sus realizaciones rítmicas difieren. 
La teoría, sin embargo, no puede meramente limitarse a afirmar esto. De la misma manera que existe un sistema de análisis en el nivel abstracto, se requiere uno en el nivel de la realización. Pero, en este caso, no es posible construir un "esquema", sino que lo que debe identificarse es una red jerárquica de regulaciones, tendencias y fenómenos circunstanciales. Más específicamente: las preguntas 2 y 3 formuladas en la sección 2 se responden en dos pasos: primero, la identificación de una serie de fenómenos del nivel de la realización, a saber, reglas (fenómenos cuya regularidad es tal que pueden considerarse esenciales para la caracterización de una manifestación del verso), tendencias (preferencias a favor o en contra de una realización determinada que el análisis estadístico revela como significativas) y hechos circunstanciales (todo lo demás). ${ }^{40}$ Segundo, debe establecerse una organización jerárquica de estos fenómenos de modo tal que se revele el modelo rítmico construido por los autores. Esta organización funciona de manera análoga a un sistema optimal de restricciones, ${ }^{41}$ en el sentido de que los poetas podían sacrificar cualquiera de sus preferencias en pos de la satisfacción de una de nivel superior. Sin embargo, la aclaración "análoga" no es vacua: a menos que uno esté dispuesto a incorporar restricciones de tipo "literarias" (e.g. la voluntad de enfatizar una determinada palabra o de dirigir la atención a un atributo de un personaje), un modelo optimal no puede abarcar nunca la complejidad del proceso compositivo de una obra de literatura. ${ }^{42}$

El modelo es difícil de ilustrar brevemente dada la complejidad de aspectos que demanda, ${ }^{43}$ pero puede ejemplificarse con el tercer verso de la estrofa sáfica, cuyo esquema es sxdsxdx. El estudio de esta estructura sugiere que presenta una única regla: la evasión de finales de la forma BLL. ${ }^{44}$ La colocación de finales de palabra después del segundo anceps (en Safo, en casi el 85\% de los casos y en Alceo, en el 90\%) podría ser considerada tanto una regla como una tendencia marcada. Algunas otras tendencias que pueden mencionarse son la realización de los ancipitia con sílabas largas (respectivamente, en 67\% y 62\% de los casos en Safo; los números en Alceo son más altos), la aparición de un final de palabras o bien en la octava o bien en la novena ubicación (después del primer o del segundo elemento de la segunda unidad s; solo hay dos excepciones seguras a esta tendencia en Safo y otras dos en Alceo, que representan menos de un 5\% de los casos) ${ }^{45}$ y la presencia de un "descenso barítono" ${ }^{46}$ en la octava ubicación de verso (en el 62\% de los versos de Safo). Dada la inevitable relación entre las últimas dos, es necesario ordenarlas jerárquicamente: ¿la preferencia por descensos en la octava ubicación es la causa o la consecuencia de la preferencia por un final de palabra en la octava o la novena ubicación? Lo segundo parece más probable que lo primero si se comparan las frecuencias de los dos fenómenos, pero se requiere un estudio detenido para demostrar que este es el caso.

Con la descripción rítmica del verso desarrollada a partir del esquema abstracto y el estudio de las preferencias de realización, el análisis de los versos particulares se enriquece. ${ }^{47}$ Así, por ejemplo, en un caso

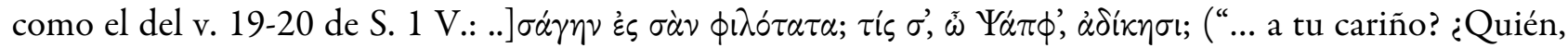
¡oh, Safo!, te agravia?”). Debe notarse la inusitada sucesión de tres monosílabos, el primero de los cuales es una de las tres palabras con final en la décima ubicación del verso, ${ }^{48}$ alargado de manera deliberada por el pronombre w, que podría haber sido colocado sin inconveniente en un lugar acaso más natural, junto al verbo. El final de palabra esperable en la undécima ubicación se debilita por la fuerte asociación entre la interjección y el nombre, que también es un monosílabo en una ubicación donde hay pocos. La secuencia está claramente diseñada para destacar cada palabra, enfatizando la pregunta ("quién") y el vocativo ("Safo"). ${ }^{49}$

La última cuestión planteada en la sección 2, la identificación de los rasgos propios de un género rítmico, que según Itsumi es excluida por Dale, puede responderse mucho mejor con el modelo delineado aquí que con las otras aproximaciones mencionadas, porque este permite discernir lo que, en cada uno, es propio de los esquemas abstractos y lo que es propio del nivel de la realización. Así, por ejemplo, la unidad ds(x), en particular en final de verso, es característica de los versos eólicos y su utilización en cualquier obra poética puede legítimamente sugerir una asociación con la técnica poética de los lesbios. ${ }^{50}$

Lo mismo puede decirse de la sucesión de dos ancipitia iniciales y, sobre todo, internos (ambos fenómenos ejemplificados en xxdsxxdsl, el metro de A. 140, entre otros), que Sicking (1993: 136) atribuye con razón a la 
sobrevivencia en el periodo de las formas con comienzo libre de varios ancipitia. Este rasgo de nivel abstracto, como se ha notado antes, ha sugerido la necesidad de incorporar como elemento ãã, es decir, la sucesión de dos ancipitia que no pueden ser realizados ambos con sílabas breves. Sin embargo, y como la propia descripción indica, la prohibición de realización kk es, justamente, del nivel de la realización, explicable, primero, por la interpretación preferida de la secuencia kk como una única ubicación métrica (en responsión con dos ubicaciones en los versos donde el par de ancipitia no se realiza de esa manera) y, segundo, si se admite la posibilidad de conservar dos ubicaciones, por la sucesión resultante de dos ubicaciones no-marcadas. Que los poetas lésbicos conserven los dobles ancipitia es muy probablemente producto de una búsqueda de variación rítmica en el comienzo de los versos (y, a veces, en su interior), que la fijación de las ubicaciones impediría.

El rasgo más importante del verso eólico, sin embargo, es la ausencia casi absoluta de divergencias rítmicas: es bien sabido que en los poetas lésbicos no hay (casi) contracción, ${ }^{51}$ resolución ni colosis. Pero en el modelo teórico colométrico (como en el de Golston y Riad y el de García Novo) este hecho es una aclaración adicional que es necesario realizar, ${ }^{52}$ no un rasgo de nivel abstracto clave en la caracterización de los esquemas métricos, como sí lo es en el presentado aquí. Lo que es más, este rasgo característico se aplica, como se ha notado, a todos los metros que produjeron los poetas eólicos (aunque, cf. n. 51, no a todos los que utilizaron).

Una ventaja adicional del modelo es que, una vez identificado el género rítmico, es posible estudiar, por así decirlo, las "especies" rítmicas, que difieren entre sí por fenómenos del nivel de la realización. ${ }^{53}$ Así, un aspecto ignorado o despreciado, como la diferencia en la distribución de palabras en Safo en una estructura como $\times \times \bar{x}$ 'd'dsx, donde hay final de palabra $34,78 \%$ de las veces antes del elemento s, y otra como $\times \times \bar{d}$ 'd'ds, donde lo mismo sucede solo en el $8,33 \%,{ }^{54}$ deja de ser un hecho menor y pasa a ser un dato significativo para entender los tipos compositivos existentes. Es acaso una distinción que podría vincularse al hecho de que una estructura tiene final sonoro y la otra final sordo (se requiere un análisis específico para demostrarlo).

\section{Conclusiones}

Dada la extensión de este trabajo, la palabra "preliminares" en su título puede resultar extraña. Sin embargo, se han dado en realidad solo dos pasos en la dirección de un nuevo modelo teórico para el estudio del metro griego antiguo. Primero, la selección de un marco provisto por los enfoques de Dale y Sicking, que no constituyen en sí mismos más que un sistema para la descripción de los metros. ${ }^{55}$ Para llegar a ese punto debieron desecharse varias teorías alternativas y, sobre todo, justificar la ya recurrentemente observada necesidad de abandonar el modelo colométrico dominante hoy.

El segundo paso ha sido la distinción de dos niveles diferentes de análisis, el de la descripción abstracta y el de la realización de los versos. En esto se ha seguido el camino abierto por Van Raalte (1986), pero se ha ido más lejos, puesto que el modelo teórico permite el estudio de todos los metros conservados. Por lo demás, si bien se ha avanzado en la descripción de cada uno de los niveles, quedan muchos detalles por considerar antes de poder empezar a hablar siquiera de una nueva teoría.

El aspecto más importante del estudio es que el modelo que este artículo pretende inaugurar no sufre de ninguna de las dificultades del actual. Esto, por supuesto, debe demostrarse en la práctica, pero parece concebible a priori que la incorporación en la enseñanza de la literatura griega antigua del esquema presentado es mucho más asequible que la incorporación de la ya inmanejablemente compleja colometría. Los elementos fundamentales son solo cuatro, las unidades básicas dos, los procesos combinatorios tres y tres también las divergencias rítmicas. Los estudiantes pueden aprender a describir con estas herramientas la inmensa mayoría de los metros conservados en poco tiempo, mientras que los aspectos propios del nivel de la realización pueden presentarse en función de los temas a estudiar. Así, mientras que en un enfoque colométrico el análisis del verso eólico demanda el aprendizaje no solo de los cola y procesos expansivos de Snell, sino de todos los términos y conceptos para analizar los metros que no pueden ser descriptos con ellos, con el modelo 
presentado aquí, los alumnos, cuya formación de base les permite reconocer sin dificultad el esquema de todos los poemas conservados, pueden aproximarse casi inmediatamente a la poesía de Safo con el conocimiento de fenómenos que en general han sido patrimonio de los especialistas y, de esta manera, comprenderla como lo que era en realidad.

\section{ReFERENCIAS}

Abritta, A. (2015) "On the Role of Accent in Ancient Greek Poetry. Pitch Patterns in the Homeric Hexameter", QUCC 111: 11-27.

Abritta, A. (2017) Hacia una historia coral de los metros griegos: Rasgos formales de los metros no-líricos desde la época arcaica hasta la antigüedad tardía, tesis de Doctorado, Buenos Aires. Recuperado de http:// repositorio.filo.uba .ar:8080/xmlui/handle/filodigital/3335.

Bassett, S. E. (1919) "The Theory of the Homeric Caesura According to the Extant Remains of the Ancient Doctrine", AJPh 40: 343-372.

Carman, C. C. (2010) "La refutabilidad del sistema de epiciclos y deferentes de Ptolomeo", Principia 14: 211-239.

Dale, A. M. (1968²) The Lyric Metres of Greek Drama, Cambridge.

Dale, A. M. (1969) Collected Papers, Cambridge.

Devine, A. M. \& Stephens, L. D. (1975) “Anceps”, GRBS 16: 197-215.

Devine, A. M. \& Stephens, L. D. (1976) “The Homeric Hexameter and a Basic Principle of Metrical Theory”, CPh 71: 141-163.

Devine, A. M. \& Stephens, L. D. (1977) "Preliminaries to an Explicit Theory of Greek Metre”, TAPA 107: 103-129.

Devine, A. M. \& Stephens, L. D. (1994) The Prosody of Greek Speech, New York.

García Novo, E. (1995) “Catalexis, Brevis in Longo and the Structure of the Greek Stichic Verse: A New Approach", RAAN 65: 73-94.

García Novo, E. (2000) "El dístico elegíaco arcaico: nueva definición de su estructura a nivel del Vers", $C F C(G)$ 10: 9-18.

García Novo, E. (2008) “Métrica griega 1984-2004”, en F. Rodríguez Adrados, J. A. Berenguer, E. R. Luján y J. Rodríguez Somolinos (eds.) Veinte años de Filología Griega (1984-2004), Madrid: 397-411.

García Novo, E. (2014) “A Structural Approach to Greek Lyrics Periods”, Rosetta 16: 1-14.

Gentili, B. \& Lomiento, L. (2003) Metrica e ritmica. Storia delle forme poetiche nella Grecia antica, Milán.

Huron, D. (2006) Sweet Anticipation. Music and the Psychology of Expectation, Cambridge.

Irigoin, J. (1956) “La structure des vers éoliens”, AC 25: 5-19.

Itsumi, K. (2009) Pindaric Metre: The 'Other Half', Oxford.

Kager, R. (1999) Optimality Theory, Cambridge.

Laguna Ortiz, A. (1997) “Nueva descripción funcional de los eolocoriambos de Alceo y Safo", $C F C(G)$ 7: 243-262.

Lorenzano, P. (2007) “Leyes fundamentales y leyes de la biología”, Scientiae Studia 5: 185-214.

Maas, P. (1962) Greek Metre, trans. Hugh Lloyd-Jones, Oxford.

Maslov, B. (2013) "The Dialect Basis of Choral Lyric and the History of Poetic Languages in Archaic Greece", SO 87: 1-29.

O'Neill Jr., E. G. (1942) “The Localization of Metrical Word Types in the Greek Hexameter”, YClS 8: 103-178.

Obbink, D. (2016) “The Newest Sappho: Text, Apparatus Criticus, and Translation”, en A. Bierl \& A. Lardinois (eds.) The Newest Sappho: P. Sapph. Obbink and P. GC inv. 105, frs. 1-4, Leiden: 13-33.

Prauscello, L. (2006) Singing Alexandria. Music Between Practice and Textual Transmission, Leiden.

Raven, D. S. (1962) Greek Metre. An Introduction, London.

Rissman, L. (1983) Love as War: Homeric Allusion in the Poetry of Sappho, Königstein. 
Rosenmeyer, P. A. (1997) “Her Master’s Voice: Sappho’s Dialogue with Homer”, MD 39: 123-149.

Ruipérez, M. (1952) “Ideas fundamentales sobre métrica griega”, EClás 1: 239-255.

Ryan, K. M. (2011) "Gradient syllable weight and weight universals in quantitative metrics", Phonology 28: 413-454.

Sicking, C. M. J. (1993) Griechische Verslehre, München.

Snell, B. (19825) Griechische Metrik, Göttingen [1º ed. 1955].

Van Ophuijsen, J. M. (1987) Hephaestion on Metre. A Translation and Commentary, Leiden.

Van Raalte, M. (1986) Rhythm and Metre. Towards a Systematic Description of Greek Stichic Verse, Assen.

Venneman, T. (1988) Preference Laws for Syllable Structure and the Explanation of Sound Change, Berlin.

Vuust, P. \& Witek, M. A. G. (2014) "Rhythmic complexity and predictive coding: a novel approach to modeling rhythm and meter perception in music", Frontiers in Psychology 5: art. 111. Recuperado de https://www.fronti ersin.org/articles/10.3389/fpsyg.2014.01111/full.

West, M. L. (1982) Greek Metre, Oxford.

\section{Notas}

1 Una observación que ya realizó Ruipérez (1952) hace medio siglo, junto con otras que el paso del tiempo ha ido verificando. En particular, un “método combinado" para el análisis del verso que utiliza la lingüística, la teoría del ritmo y la métrica comparada como el que el autor propone (p. 251) ha sido, aunque no siempre con el reconocimiento debido, la fuente de mayores avances en la metricología de las últimas décadas.

2. Omito, dado que esta presentación incluye solo consideraciones analíticas, el problema de que el testimonio antiguo de hecho no es una fuente demasiado confiable sobre la forma en que los compositores elaboraban sus versos (cf. e. g. Van Ophuijsen, 1987: 27, sobre el objetivo de la metricología antigua). El estado de nuestro conocimiento es tan pobre que ni siquiera podemos estar del todo seguros sobre el grado de sofisticación técnica (entiéndase: el grado de conciencia de la propia sofisticación técnica) que tenían los poetas líricos arcaicos, un problema que se ejemplifica en el debate sobre la utilidad (y hasta la existencia) del concepto de colon en la época pre-helenística (cf. Prauscello, 2006: 7-8, n. 4 y el todavía importante Bassett, 1919).

3. Un ejemplo relativamente reciente de esta tendencia en Itsumi (2009: 7): "We should not prematurely give up hope that a consistent, objective colometry may be available for Pindaric metres as well as for other Greek poetry". Resulta difícil de entender la noción de "prematuramente" aplicada por el autor a un debate que lleva más de cien años.

4. Cf. Devine y Stephens (1977) y Sicking (1993: 9-16), ambos (en particular el primero) con críticas contundentes e insalvables al enfoque.

5. Utilizo "tradicional” en el sentido de Devine y Stephens (1977), esto es, para referirme a teorías donde los únicos valores métricos que cuentan son "larga" y "breve".

6. Una vez más, una realidad que preocupaba ya a Ruipérez (1952: 239-240) y la metricología ha hecho poco por modificar.

7. Una oposición que está implicada en la clásica entre “métrica interna” y “métrica externa” de O’Neill (1942: 105, n. 2) y que puede resultar en cierto modo contra-intuitiva (“¿no están las palabras constituidas por sílabas y no están las sílabas en palabras?”). No obstante, en el análisis del verso es bastante claro que hay fenómenos propios del nivel de la distribución silábica (e.g. la frecuencia de realizaciones de $e . g$. un elemento anceps) y otros propios del nivel de la distribución de palabras (la evasión de finales de palabra en determinadas ubicaciones, los llamados "puentes"). Por supuesto, también hay fenómenos que cruzan ambos niveles (e.g. los “puentes prosódicos” en Snell, 1982: 11-12).

8. Sobre la necesidad de distinguir niveles de análisis, pocas veces tomada en consideración en el estudio del verso griego, cf. Ruipérez (1952: 248-249) y, sobre todo, Van Raalte (1986: 1-13). No utilizaré la terminología de la autora (métrico vs. rítmico) porque me resulta algo confusa y, además, poco apropiada para una diferenciación metodológico-teórica, no en el objeto de estudio.

9. Esto es porque el autor propone encarar el verso griego como una sucesión de ubicaciones con rangos de tolerancia a un conjunto de valores duracionales. La primera sílaba del endecasílabo sáfico no sería así un longum (ver abajo), sino una ubicación que tolera sílabas internas con vocal larga, sílabas finales con vocal larga, sílabas con vocal breve cerradas por una consonante, etc. Aunque esta propuesta suele ser considerada "extrema", es importante observar que en realidad lo único extremo es la cantidad de tipos silábicos que West introduce sin justificación: cualquier enfoque duracionalista interpreta todas las ubicaciones como rangos de tolerancia. 
10. Las razones que da García Novo para distinguir entre un enfoque como el de Gentili y Lomiento (2003) y uno como el de Maas (1962) o West (1982) son atendibles pero superficiales. Como se señalará a continuación, todas estas aproximaciones se caracterizan por comprimir niveles de análisis y pueden ser incluidas en el campo de la colometría.

11. El hecho de que el segundo haya sido publicado después del primero se presta a confusión, pero Irigoin mismo (1956: 19) informa al final del artículo que su trabajo fue escrito antes de la publicación del texto de Snell.

12. Un vistazo por el conspectum metrorum de la edición de Voigt basta para demostrar esto. 18 de 31 de las estructuras métricas sáficas (contando todas las mencionadas por Voigt y cada estrofa como una única estructura) no son "eólicas" y 12 de 39 de las de Alceo. Incluso ponderando los usos efectivos de los poetas, aunque la cantidad de metros "no-eólicos" disminuye mucho, sigue siendo considerable.

13. El número surge de la multiplicación de los seis cola fundamentales mencionados en las dos preguntas anteriores por las siete formas en que pueden presentarse: puros, con expansión dactílica simple, doble o triple, con expansión coriámbica simple, doble o triple.

14. Una observación que se encuentra en Ruipérez (1952: 241): “(..) este tipo de Métrica procedente de la Antigüedad y cultivado rutinariamente hasta nuestros días (...) no puede aspirar más que a una descripción mecánica con fines mnemónicos. (...) Pero hay que tener presente que esta Métrica no explica nada (...) y que las etiquetas que emplea son de un manejo sumamente peligroso, porque con ellas se corre el riesgo de que, como de hecho sucede, se tome por una interpretación rítmica e incluso genética lo que solo es una descripción externa".

15. Volveré sobre esto más adelante, pero nótese que lo que caracteriza a un enfoque duracionalista es precisamente la premisa de que las oposiciones cuantitativas de estructura silábica deben ser incorporadas en la descripción de nivel abstracto de los metros. La alternativa mencionada en la sección 2 de que en el nivel de la realización haya algo más que sílabas largas y breves no es "duracionalista" (al menos no en sentido estricto).

16. Sobre el sistema ptolemaico, su carácter complejo y su posibilidad de refutación, cf. Carman (2010).

17. El trabajo de Itsumi (2009) ha sido clave en este proceso por su sistematización de los metros eólicos en Píndaro. Sin embargo, es también el colmo de la complicación del modelo teórico en una medida que basta para hacerlo inadmisible.

18. La trilogía fue reimpresa en Dale (1969: 41-97).

19. Sobre el cual cf. Maas (1962: 40-42); recuérdese que la Griechische Metrik fue publicada originalmente en 1923.

20. Dejo de lado las que corresponden al largo de las líneas, haciendo notar, como ya he hecho en otro lugar, que ninguna teoría métrica ha propuesto explicaciones para este importante aspecto de los versos. Las hipótesis de Golston y Riad, por ello y a pesar de su inefectividad en los metros líricos, resultan de particular importancia.

21. Siguiendo el análisis de Devine y Stephens (1994), Golston y Riad consideran estresadas todas las sílabas largas y la primera breve de las secuencias BB.

22. En una sílaba larga, los autores entienden que la mora estresada es la primera.

23. Esto se hace evidente en el hecho de que ni Píndaro ni los coros dramáticos son incorporados en el análisis de Golston y Riad, acaso por la simple razón de que no toleran el enfoque sin numerosas restricciones adicionales. Otra objeción importante al enfoque abajo, n. 52 .

24. Sobre las cuales cf. Golston y Riad (2005: 109-110).

25. La separación del esquema abstracto y las realizaciones de un verso no puede justificar ignorar por completo la realización a la hora de reconstruir el esquema abstracto. ¿Qué clase de elemento “potencialmente disilábico” es, por ejemplo, el quinto longum del trímetro, que en el yambo arcaico y en la tragedia resuelve una vez cada varios miles de versos y en no pocas obras nunca? La afirmación (García Novo, 1995: 82, n. 41) de que los componentes D realizados por doble breve son necesarios cada tanto para "underline the divisible nature of those components" no solo sugiere que el esquema del trímetro varía en función de qué resoluciones se dan en cada poema (en una tragedia, como muchas, donde varios de los longa no resuelven nunca, debería postularse un esquema con varios $\mathrm{M}^{1}$ ), sino que además es sencillamente falsa: en los trímetros conservados de Semónides, por ejemplo, no hay un solo caso de resolución en ninguna ubicación.

26. Omito aquí discutir algunos aspectos puntuales que son importantes para la autora, como el (justificado) abandono del concepto de "catalexis" y la reinterpretación del principio brevis in longo.

27. Entiendo que esta podría ser considerada la "ley fundamental" de la metricología, en el sentido de "ley fundamental" expresado en Lorenzano (2007): $x$ es un metro si $x$ es una sucesión limitada de ubicaciones métricas. Como puede verse, el principio vale (cuasi-vacuamente, como suele suceder con las leyes fundamentales) para todos los metros humanos concebibles. Nótese, asimismo, que el concepto "ubicación métrica” es teórico (en el sentido estructuralista de que para delimitar el concepto es necesaria la teoría o, en palabras simples, por el mero hecho de que "ubicación métrica" solo puede tener sentido en el marco de un modelo métrico).

28. Una posibilidad de hecho propuesta en ocasiones, por ejemplo West (1982: 31), que afirma que en el verso eólico alternan formas regulares y anaclásticas (contra -típicamente sin explicitarlo-Snell, 1982: 47, que afirma con toda razón que no hay casos ciertos de ese fenómeno). Nótese, no obstante, que esta admisión de alternancia no es más que una 
solución al problema específico que presenta una estructura de cierta dificultad, no una premisa metodológica de mucho alcance.

29. Formalmente, anceps debe definirse como "elemento no-marcado variable", por razones sobre las que no me puedo extender aquí. A los fines de la presente exposición, basta con entender el anceps como la ubicación métrica en cuya realización pueden hallarse una sílaba larga o una sílaba breve.

30. Las únicas ubicaciones métricas marcadas son los longa, siguiendo el principio universal de que, en lenguas cuantitativas, las sílabas largas son prominentes (Venneman, 1988).

31. Sobre lo primero, la evidencia reciente (Ryan, 2011) contradice en parte la presentada por Devine y Stephens (1975, 1976 y 1977), pero, debe notarse, esto no afecta en absoluto la cuestión, que, dadas las múltiples interpretaciones posibles de los resultados (cf. https://www.researchgate.net/post/How_is_the_concept_of_V-toV_different_from_other_durational_approaches_to_Ancient_Greek_Poetry), debe definirse en el nivel meta-teórico. Lo segundo es más complejo, porque de ser cierto efectivamente demandaría teorías métricas diferentes a las propuestas aquí, pero la cantidad despreciable de evidencia que lo sugiere no basta para invertir la carga de la prueba (cf. Sicking, 1993: 32-37).

32. Esta afirmación presupone que, con los cuatro elementos mencionados arriba, pueden describirse la totalidad de los metros griegos. A priori, por supuesto, esto no es necesariamente cierto, pero se verifica en la práctica.

33. El concepto ha sido introducido en Van Raalte (1986: 13-15).

34. En este punto se hace evidente la necesidad de introducir términos para referirse a los elementos de nivel abstracto. Si se quisiera evadir el uso de e. g. longum, la posibilidad de la resolución forzaría a hablar de una sílaba larga que puede realizarse con dos sílabas breves, lo que inevitablemente llevaría a confusiones.

35. Un principio para el que he utilizado en discusiones específicas el término brevis in longo, que en rigor significa otra cosa pero que, estando estandarizado, puede resignificarse sin inconvenientes. Utilizo, como se habrá notado, el signo de anceps para la ubicación final, que resulta menos confusa a mi entender que la utilización más habitual del signo para longum (“-"). Nótese, no obstante, que, como han observado entre otros Devine y Stephens (1975: 198), el anceps propiamente dicho y la ubicación brevis in longo son elementos de naturaleza diferente.

36. Por supuesto, he dejado de lado algunas consideraciones adicionales importantes. Recuérdese que este estudio es solo preliminar.

37. Omito una consideración especial de estos dos grupos de elementos, en donde deben incluirse varias clases diferentes, dependiendo de los resultados de los análisis. Diferencias cuantitativas sub-moraicas (del tipo preferido por los duracionalistas), por ejemplo, que no tienen lugar en el nivel de la descripción abstracta, pueden ser tomadas en consideración por los poetas en el nivel de la realización. Lo mismo puede decirse de la distinción entre palabras ortotónicas y clíticos, entre sílabas acentuadas y no-acentuadas, etc.

38. El análisis estadístico del verso no es solo una conveniencia metodológica o una demanda de "objetividad" en los estudios de metricología, sino que responde a la forma en que los seres humanos de hecho percibimos el ritmo y la música (cf. Huron, 2006: passim y esp. 73-131) y Vuust y Witek (2014), que, siguiendo una tendencia extendida en la psicología reciente, proponen un modelo bayesiano (i.e. probabilístico) para la percepción del ritmo musical.

39. Como Sicking (1993: 59-60 y 214), entiendo que los nombres tradicionales de algunas secuencias, aunque carezcan de valor teórico, tienen un cierto valor instrumental.

40. Estas tres clases de fenómenos no son cualitativamente diferentes, sino solo cuantitativamente diferentes. Lo que en un determinado autor es una tendencia puede ser una regla en otro y un hecho circunstancial en un tercero.

41. Cf. entre otras muchas posibles referencias Kager (1999). La idea es, por supuesto, afín a la propuesta por Golston y Riad, con la salvedad de que, mientras ellos entienden que el metro debe ser descripto únicamente de esta manera, yo he restringido el modelo basado en restricciones al nivel de la realización. Entiendo que esto es más adecuado, en particular en una tradición poética donde los esquemas métricos tienen orígenes muy diversos y no pocas veces ajenos por completo al lenguaje (por lo menos en su forma contemporánea al verso).

42. Dos objeciones se pueden presentar a esta observación: primero, ¿por qué no incorporar restricciones de tipo "literario"? En principio, no hay ninguna buena respuesta. Invito al lector, no obstante, a intentar elaborar esas restricciones sin convertir la teoría en una madeja inmanejable de instrumentos ad hoc. La segunda objeción tiene que ver con la introducción del "proceso compositivo" en este punto, que se ha reiterado no debe ser tomado en cuenta en la elaboración de las teorías métricas. Lo dicho no va en detrimento de eso: nótese que es justamente porque en poesía entran en juego aspectos diferentes al métrico que aquí he introducido la noción de "proceso compositivo".

43. Sin ir más lejos, el libro completo de Van Raalte (1986) no es más que un estudio de preferencias de realización en los metros estíquicos griegos.

44. Me encuentro preparando un trabajo para demostrar esto.

45. La frecuencia exacta no es sencilla de definir porque depende de qué versos uno esté dispuesto a conservar en la

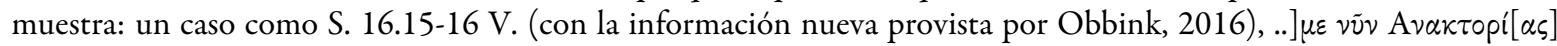

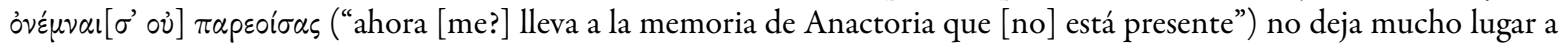
dudas de 
que hay una palabra con final allí, por lo que, aunque esté perdida la última sílaba, no puede dejarse de lado, pero en A.

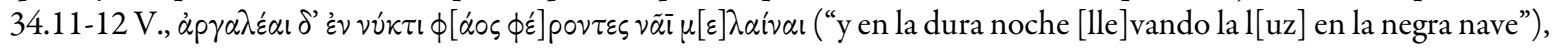

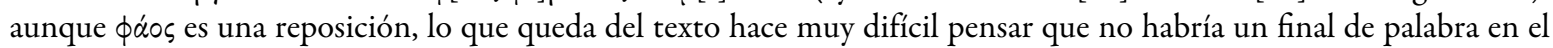
espacio perdido. El problema principal, obsérvese, no es tanto si había o no había final de palabra como si el verso debe o no ser incluido en la muestra de análisis.

46. Cf. Abritta (2017: 56-59).

47. Nótese, recordando lo dicho en la n. 38 , que este enriquecimiento no solo mejora nuestras lecturas, sino que las acerca más a la experiencia efectiva de los oyentes antiguos, que reconocerían los fenómenos del nivel de la realización sobre la base de su frecuencia estadística, ya sea esperando los previsibles o sorprendiéndose ante los imprevisibles. Dado que los poetas indudablemente utilizarían y manipularían en sus composiciones ese reconocimiento, no es admisible encarar el estudio de su poesía sin intentar tomarlo en cuenta.

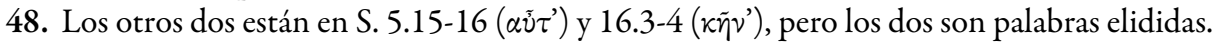

49. Es interesante observar que este recurso se refuerza por la distribución de acentos: las dos palabras enfatizadas por su ubicación ( $\tau$ í y $\left.\Psi^{\prime} \alpha \dot{\alpha} \pi \phi^{\prime}\right)$ son también las únicas con una prominencia oxítona, $\tilde{\omega}$ es el único caso conservado de circunflejo en undécima ubicación y toda la oración cierra con una forma proparoxítona con una penúltima sílaba prominente con tono descendente. Cf. sobre esta interpretación de las marcas acentuales y su importancia en el verso griego, Abritta (2015 y 2017).

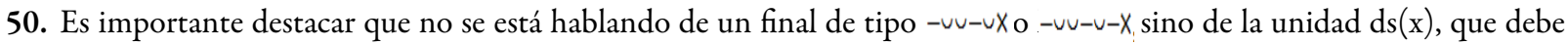
poder reconocerse por yuxtaposición o separación con anceps. Esto sucede, por ejemplo, en Esq., P.V. 128-135, un pasaje reconocido como "eólico" en e.g. Dale (1968: 163). El quinto verso de la primera estrofa del párodo de Sóf., O.T. (vv. 151-167) sirve como contraejemplo: aunque termina con la secuencia ds, esta no constituye una unidad sino el final de una tirada dactílica que atraviesa toda la línea, cuyo esquema es ddddddddds (cf. Sicking, 1993: 200-201) y por supuesto no tiene afinidad alguna con el verso eólico.

51. Las únicas excepciones son los hexámetros que aparecen en el corpus, como en S. 105 o 106 V. Dada la influencia de Homero que se percibe en Safo y Alceo (cf. e. g. Rissman, 1983 y, en general sobre el problema de las alusiones en Safo, Rosenmeyer, 1997), no puede sorprender que hayan incursionado ocasionalmente en un metro extranjero para ellos (un fenómeno paralelo a la incorporación de formas jónicas en su poesía producida en dialecto literario proto-eólico, de acuerdo con el análisis de Maslov, 2013). Es, sin lugar a dudas, mucho más fácil de explicar que el hecho de que hayan compuesto una gran parte de su poesía en un estilo y otra parte en otro (cf. sec. 3).

52. El fenómeno es particularmente problemático en el enfoque de Golston y Riad, dado que su premisa fundamental es que la alternancia -v/--y -vv/-- es más básica que la fijación rítmica de una u otra unidad. El hecho de que en la tradición poética griega exista un género como el eólico, donde no hay tales alternancias, hace de su modelo uno menos adecuado que cualquiera de los otros, en donde estas son un fenómeno posterior.

53. Y, continuando con la analogía, se podría incluso hablar de "razas" métricas al estudiar las diferencias de realización entre dos corpora que utilizan el mismo esquema.

54. Los números concretos son los siguientes: en $\times \mathrm{d}^{\prime} \mathrm{d}$ 'ds $\times 16$ de 46 versos conservados tienen pausa en la ubicación anterior a s, mientras que en $\times \times d^{\prime} d$ 'ds, 1 solo de 12 (el v. 56.1 V.). El cálculo se realiza solo tomando en cuenta palabras métricas (esto es, excluyendo proclíticos - un caso en 57.3 V.- y palabras seguidas de enclítico - un caso, quizás discutible, en 56.2 V.-).

55. Entiéndase: a los fines del presente trabajo. Los autores hacen importantes observaciones que es necesario tomar en cuenta en el análisis rítmico que aquí he debido dejar de lado.

* Doctor en Letras Clásicas, Licenciado en Letras y Licenciado en Filosofía por la Universidad de Buenos Aires. Actualmente es becario posdoctoral del Conicet y su área de investigación es la métrica griega antigua. Ha publicado artículos en revistas nacionales e internacionales sobre estos temas y también sobre la himnodia hexamétrica en la Grecia

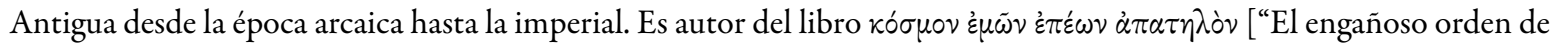
mis palabras"]: Un análisis coral del poema de Parménides, con traducción y comentario textual (FFyL-UBA, en prensa). 